International Journal of Modern Physics C

(C) World Scientific Publishing Company

\title{
Preperiodicity and systematic extraction of periodic orbits of the quadratic map
}

\author{
Jason A.C. Gallas \\ Instituto de Altos Estudos da Paraíba, Rua Silvino Lopes 419-2502, \\ 58039-190 João Pessoa, Brazil, \\ Complexity Sciences Center, 9225 Collins Ave. Suite 1208, Surfside FL 33154, USA, \\ Max-Planck-Institut für Physik komplexer Systeme, 01187 Dresden, Germany \\ jason.gallas@gmail.com \\ Received 21 August 2020 \\ Accepted 27 August 2020 \\ https://doi.org/10.1142/S0129183120501740
}

\begin{abstract}
Iteration of the quadratic map produces sequences of polynomials whose degrees explode as the orbital period grows more and more. The polynomial mixing all 335 period- 12 orbits has degree 4020 , while for the 52,377 period-20 orbits the degree rises already to $1,047,540$. Here, we show how to use preperiodic points to systematically extract exact equations of motion, one by one, with no need for iteration. Exact orbital equations provide valuable insight about the arithmetic structure and nesting properties of towers of algebraic numbers which define orbital points and bifurcation cascades of the map.

Keywords: Arithmetic dynamics; Preperiodic points; Quadratic map; Exact orbital equations.
\end{abstract}

PACS Nos.: 02.10.De, 03.65.Fd, 02.70.Wz

\section{Introduction}

A recent paper in this Journa ${ }^{11}$ described an iterative approach to detect orbit within orbit stratification in the so-called $a=2$ partition generating limit $t^{2 / 4}$ of the quadratic or, equivalently, logistic map

$$
x_{t+1} \equiv f\left(x_{t}\right)=a-x_{t}^{2}, \quad t=0,1,2, \ldots
$$

Orbit within orbit stratification means that periodic orbits are not always necessarily independent of each other. As discussed in Refs $\frac{115}{15}$, apart from the intrinsic interest in detecting interdependent orbits, stratification is potentially significant to, e.g., rearrange trajectories sums in trace formulas underlying a semiclassical interpretation of atomic physics spectra.

The existence of stratification was originally detected by extracting orbits from polynomials of very high degrees obtained by iterating Eq. (11). However, map iteration generates sequences of mammoth polynomials whose degrees explode as the orbital period grows more and more. To bypass this difficulty it was conjectured that exploration of preperiodic points could provide a viable alternative to iteration.

Here, the aim is to complement the work of Ref ${ }^{1}$ by introducing and explicitly implementing an alternative algorithm based on preperiodic points. As it is known, $\frac{6}{6}$ for $a=2$ preperiodic points are easy to obtain. They are roots of an infinite family of polynomials $Q_{\ell}(x)$ generated by a recursive relation, given below in Eq. (6). Starting from preperiodic points one quickly lands in periodic cycles. So far, preperiodic points were used ${ }^{\sqrt{6}}$ to extract specific orbital equations embedded in polynomial clusters with degree exceeding one billion and, consequently, totally out of reach by ordinary brute-force polynomial factorization. Here, in contrast, the aim is to 
investigate whether or not the roots of $Q_{\ell}(x)$ are able to generate systematically, one by one, all orbits of the map. We find that they are, as described in what follows.

\section{Motivation to perform exact analytical work}

For any given period $k$, equations of motion are defined by $k$-degree polynomials which have either integer or algebraic numbers as coefficients. Thus, the algebraic nature of equations of motion depends of the algebraic character of the polynomial coefficients representing the orbit. By way of illustration, consider the following triplet of exact period-five orbits! 1

$$
\begin{aligned}
& o_{5,1}(x)=x^{5}-x^{4}-4 x^{3}+3 x^{2}+3 x-1, \\
& o_{5,2}(x)=x^{5}+\frac{1}{2}(1+\sqrt{33}) x^{4}-x^{3}-\frac{3}{2}(3+\sqrt{33}) x^{2}-(6+\sqrt{33}) x-1, \\
& o_{5,3}(x)=x^{5}+\frac{1}{2}(1-\sqrt{33}) x^{4}-x^{3}-\frac{3}{2}(3-\sqrt{33}) x^{2}-(6-\sqrt{33}) x-1 .
\end{aligned}
$$

Technically, $o_{5,2}(x)$ and $o_{5,3}(x)$ are conjugated over the quadratic number field $\mathbb{Q}(\sqrt{33})$. When multiplied together, they produce an orbital cluster $c_{5,1}(x)$, namely

$$
\begin{aligned}
c_{5,1}(x) & =o_{5,2}(x) \cdot o_{5,3}(x), \\
& =x^{10}+x^{9}-10 x^{8}-10 x^{7}+34 x^{6}+34 x^{5}-43 x^{4}-43 x^{3}+12 x^{2}+12 x+1 .
\end{aligned}
$$

Manifestly, this orbital aggregate has integer coefficients and, therefore, is arithmetically simpler than the pair of orbits that it contains. While to multiply known conjugate orbits is an easy task, the inverse problem, to disentangle conjugated orbits from a given cluster, is a quite hard problem, particularly for aggregates of orbits with odd or high degrees. Iteration produces a profusion of orbital aggregates that need to be disentangled.

Notice that since it is not possible to represent $\sqrt{33}$ numerically without truncation, the coefficients in $o_{5,2}(x)$ and $o_{5,3}(x)$ can only be represented numerically as approximations. This means that numerical (inexact) work precludes recognizing orbital conjugations such as the symmetric decompositions clearly visible between $o_{5,2}(x)$ and $o_{5,3}(x)$. Nevertheless, as shown below, knowing that orbital conjugation exists one can suitably sift and multiply inexact orbits, searching for expressions which turn out to have nearly integer coefficients. This is the key idea to be explored in the remainder of the paper.

As mentioned, the big challenge is to derive exact expressions for orbits when the orbital period $k$ grows without bound. A complication hampering such derivation is that current computer algebra systems are essentially adapted to deal with procedures developed for integers, not for generic algebraic numbers of arbitrary degrees, a considerably harder problem. This motivates pursuing the alternative procedure discussed here, which profits from preperiodic points of the $Q_{\ell}(x)$ family.

\section{Generation of the $Q_{\ell}(x)$ polynomials}

The $Q_{\ell}(x)$ polynomials are obtained as irreducible factors of an auxiliary family of polynomials, $T_{\ell}(x)$, which are generated recursively. Starting from two initial seed functions, $T_{0}(x)$ and $T_{1}(x)$, subsequent $T_{\ell}(x)$ are obtained from the recurrence $e^{7}$

$$
T_{\ell}(x)=x T_{\ell-1}(x)-T_{\ell-2}(x), \quad \ell=2,3,4, \ldots
$$

For our present purpose, we fix $T_{0}(x)=2$ and $T_{1}(x)=x$. Instead of the recurrence, a direct way to obtain $T_{\ell}(x)$ is from Pincherle's relation ${ }^{8}$

$$
T_{\ell}(x)=\left(\frac{x-\sqrt{x^{2}-4}}{2}\right)^{\ell}+\left(\frac{x+\sqrt{x^{2}-4}}{2}\right)^{\ell}, \quad \ell=0,1,2, \ldots
$$


October 9, $2020 \quad$ 0:27 WSPC/INSTRUCTION FILE Qpols for arxiv

Preperiodicity and systematic extraction of periodic orbits of the quadratic map 3

Table 1. Characteristics of the orbits produced by the roots of the first 120 polynomials $Q_{\ell}(x)$. The label $\ell$ refers to $Q_{\ell}(x)$ while $\partial_{\ell}$ gives the degree of the $Q_{\ell}(x)$ which has the shortest preperiodic transient. Here, $l$ is the length of the preperiodic transient leading to the orbit, and $k$ is the orbital period.

\begin{tabular}{|c|c|c|c|c|c|c|c|c|c|c|c|c|c|c|}
\hline $\bar{i}$ & $\bar{\ell}$ & $\partial_{\ell}$ & 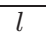 & $\bar{k}$ & $\bar{i}$ & $\ell$ & $\partial \ell$ & $l$ & $k$ & $i$ & $\ell$ & $\partial_{\ell}$ & l & $k$ \\
\hline 1 & 2 & 2 & 3 & 1 & 41 & 120 & 64 & 5 & 4 & 81 & 258 & 168 & 3 & 7 \\
\hline 2 & 3 & 2 & 2 & 1 & 42 & 136 & 128 & 5 & 4 & 82 & 254 & 252 & 3 & 7 \\
\hline 3 & 4 & 4 & 4 & 1 & 43 & 240 & 128 & 6 & 4 & 83 & 344 & 336 & 5 & 7 \\
\hline 4 & 6 & 4 & 3 & 1 & 44 & 272 & 256 & 6 & 4 & 84 & 51 & 32 & 2 & 8 \\
\hline 5 & 8 & 8 & 5 & 1 & 45 & 11 & 10 & 2 & 5 & 85 & 85 & 64 & 2 & 8 \\
\hline 6 & 12 & 8 & 4 & 1 & 46 & 22 & 20 & 3 & 5 & 86 & 102 & 64 & 3 & 8 \\
\hline 7 & 16 & 16 & 6 & 1 & 47 & 33 & 20 & 2 & 5 & 87 & 170 & 128 & 3 & 8 \\
\hline 8 & 24 & 16 & 5 & 1 & 48 & 31 & 30 & 2 & 5 & 88 & 255 & 128 & 2 & 8 \\
\hline 9 & 32 & 32 & 7 & 1 & 49 & 44 & 40 & 4 & 5 & 89 & 257 & 256 & 2 & 8 \\
\hline 10 & 48 & 32 & 6 & 1 & 50 & 66 & 40 & 3 & 5 & 90 & 340 & 256 & 4 & 8 \\
\hline 11 & 64 & 64 & 8 & 1 & 51 & 62 & 60 & 3 & 5 & 91 & 408 & 256 & 5 & 8 \\
\hline 12 & 96 & 64 & 7 & 1 & 52 & 88 & 80 & 5 & 5 & 92 & 19 & 18 & 2 & 9 \\
\hline 13 & 128 & 128 & 9 & 1 & 53 & 132 & 80 & 4 & 5 & 93 & 27 & 18 & 2 & 9 \\
\hline 14 & 192 & 128 & 8 & 1 & 54 & 124 & 120 & 4 & 5 & 94 & 38 & 36 & 3 & 9 \\
\hline 15 & 256 & 256 & 10 & 1 & 55 & 176 & 160 & 6 & 5 & 95 & 54 & 36 & 3 & 9 \\
\hline 16 & 5 & 4 & 2 & 2 & 56 & 264 & 160 & 5 & 5 & 96 & 57 & 36 & 2 & 9 \\
\hline 17 & 10 & 8 & 3 & 2 & 57 & 248 & 240 & 5 & 5 & 97 & 73 & 72 & 2 & 9 \\
\hline 18 & 20 & 16 & 4 & 2 & 58 & 352 & 320 & 7 & 5 & 98 & 76 & 72 & 4 & 9 \\
\hline 19 & 40 & 32 & 5 & 2 & 59 & 13 & 12 & 2 & 6 & 99 & 108 & 72 & 4 & 9 \\
\hline 20 & 80 & 64 & 6 & 2 & 60 & 21 & 12 & 2 & 6 & 100 & 114 & 72 & 3 & 9 \\
\hline 21 & 160 & 128 & 7 & 2 & 61 & 26 & 24 & 3 & 6 & 101 & 171 & 108 & 2 & 9 \\
\hline 22 & 320 & 256 & 8 & 2 & 62 & 42 & 24 & 3 & 6 & 102 & 146 & 144 & 3 & 9 \\
\hline 23 & 7 & 6 & 2 & 3 & 63 & 63 & 36 & 2 & 6 & 103 & 152 & 144 & 5 & 9 \\
\hline 24 & 9 & 6 & 2 & 3 & 64 & 52 & 48 & 4 & 6 & 104 & 216 & 144 & 5 & 9 \\
\hline 25 & 14 & 12 & 3 & 3 & 65 & 65 & 48 & 2 & 6 & 105 & 228 & 144 & 4 & 9 \\
\hline 26 & 18 & 12 & 3 & 3 & 66 & 84 & 48 & 4 & 6 & 106 & 342 & 216 & 3 & 9 \\
\hline 27 & 28 & 24 & 4 & 3 & 67 & 126 & 72 & 3 & 6 & 107 & 304 & 288 & 6 & 9 \\
\hline 28 & 36 & 24 & 4 & 3 & 68 & 104 & 96 & 5 & 6 & 108 & 432 & 288 & 6 & 9 \\
\hline 29 & 56 & 48 & 5 & 3 & 69 & 130 & 96 & 3 & 6 & 109 & 437 & 396 & 86 & 9 \\
\hline 30 & 72 & 48 & 5 & 3 & 70 & 168 & 96 & 5 & 6 & 110 & 25 & 20 & 2 & 10 \\
\hline 31 & 112 & 96 & 6 & 3 & 71 & 252 & 144 & 4 & 6 & 111 & 41 & 40 & 2 & 10 \\
\hline 32 & 144 & 96 & 6 & 3 & 72 & 208 & 192 & 6 & 6 & 112 & 50 & 40 & 3 & 10 \\
\hline 33 & 224 & 192 & 7 & 3 & 73 & 260 & 192 & 4 & 6 & 113 & 93 & 60 & 2 & 10 \\
\hline 34 & 288 & 192 & 7 & 3 & 74 & 336 & 192 & 6 & 6 & 114 & 82 & 80 & 3 & 10 \\
\hline 35 & 15 & 8 & 2 & 4 & 75 & 416 & 384 & 7 & 6 & 115 & 100 & 80 & 4 & 10 \\
\hline 36 & 17 & 16 & 2 & 4 & 76 & 43 & 42 & 2 & 7 & 116 & 164 & 160 & 4 & 10 \\
\hline 37 & 30 & 16 & 3 & 4 & 77 & 86 & 84 & 3 & 7 & 117 & 200 & 160 & 5 & 10 \\
\hline 38 & 34 & 32 & 3 & 4 & 78 & 129 & 84 & 2 & 7 & 118 & 205 & 160 & 2 & 10 \\
\hline 39 & 60 & 32 & 4 & 4 & 79 & 127 & 126 & 2 & 7 & 119 & 341 & 300 & 2 & 10 \\
\hline 40 & 68 & 64 & 4 & 4 & 80 & 172 & 168 & 4 & 7 & 120 & 328 & 320 & 5 & 10 \\
\hline
\end{tabular}

For $\ell=1, T_{1}(x)=Q_{1}(x)=x$. For $\ell>1$, the polynomials $T_{\ell}(x)$ are always given by products of cyclotomic-like irreducible factors $Q_{\ell}(x)$, except for $\ell=2^{n}, n=1,2,3, \ldots$ when $T_{\ell}(x)=Q_{\ell}(x)$. Every new $T_{\ell}(x)$ generated by Eq. (6) contributes a new irreducible factor $Q_{\ell}(x)$, new in the sense of not appearing for any index $\ell^{\prime}$ smaller than $\ell$. Thus, the first few are $T_{1}(x)=Q_{1}(x)=x, T_{2}(x)=Q_{2}(x)=x^{2}-2$, and

$$
T_{3}(x)=Q_{1}(x) Q_{3}(x), \quad T_{4}(x)=Q_{4}(x), \quad T_{5}(x)=Q_{1}(x) Q_{5}(x), \quad T_{6}(x)=Q_{2}(x) Q_{6}(x),
$$

where

$$
Q_{3}(x)=x^{2}-3, \quad Q_{4}(x)=x^{4}-4 x^{2}+2, \quad Q_{5}(x)=x^{4}-5 x^{2}+5, \quad Q_{6}(x)=x^{4}-4 x^{2}+1 .
$$

The first twenty $T_{\ell}(x)$ and $Q_{\ell}(x)$ are listed in Table 1 of an open access paper ${ }^{6}$ The key observation is that the irreducible $Q_{\ell}(x)$ are the building blocks of the reducible auxiliary $T_{\ell}(x)$. 
Using the roots of $Q_{\ell}(x)$ as starting conditions to iterate the quadratic map, Eq. (1), one finds that after a preperiodic start, i.e. a certain number of non-repeating iterates, the iteration lands on a cycle of $k$ distinct points that repeats forever.

\section{The selective extraction of periodic orbits}

Using Eq. (6) we generated the first 400 polynomials $T_{\ell}(x)$ and $Q_{\ell}(x)$. Irreducible $T_{\ell}(x)$ imply $Q_{\ell}(x)=T_{\ell}(x)$. Otherwise, $Q_{\ell}(x)$ is the factor of highest degree in $T_{\ell}(x)$. Therefore, while the degree of $T_{\ell}(x)$ grows steadily with $\ell$, the degree of $Q_{\ell}(x)$ fluctuates, i.e. emerges not in a regular order. After generating the $400 Q_{\ell}(x)$ polynomials, we investigated in which periodic orbit their roots land. Clearly, there are several roots to choose as initial conditions of the iterative process. Thus, in addition to roots leading to genuine period- $k$ orbits one may also find roots leading to orbits of smaller periods, divisors of $k$.

Table 1 illustrates data for 120 of the 400 polynomials, ordered according the period $k$. This table reveals interesting systematic patterns and trends which will be considered in more detail now.

\subsection{Preperiodic generation of period four orbits and aggregates}

As it is known, there are three possible period-four orbits for the quadratic map, namely

$$
\begin{aligned}
o_{4,1}(x) & =x^{4}+x^{3}-4 x^{2}-4 x+1, \\
o_{4,2}(x) & =x^{4}-\frac{1}{2}(1-\sqrt{17}) x^{3}-\frac{1}{2}(3+\sqrt{17}) x^{2}-(2+\sqrt{17}) x-1, \\
& \simeq x^{4}+1.561553 x^{3}-3.561553 x^{2}-6.123106 x-1, \\
o_{4,3}(x) & =x^{4}-\frac{1}{2}(1+\sqrt{17}) x^{3}-\frac{1}{2}(3-\sqrt{17}) x^{2}-(2-\sqrt{17}) x-1, \\
& \simeq x^{4}-2.561553 x^{3}+0.561553 x^{2}+2.123106 x-1 .
\end{aligned}
$$

Here, we have also indicated approximate "projections" onto the real axis for two orbits. This was done to emphasize that, since no exact representation onto the real axis is possible for $\sqrt{17}$, independently of the number of digits used, such projections will be necessarily just approximations of the exact equations of motions, obliterating completely the conjugation symmetry between $o_{4,2}(x)$ and $o_{4,3}(x)$. Nevertheless, knowing that $o_{4,2}(x)$ and $o_{4,3}(x)$ are conjugated naturally lead us to multiply them together to obtain an approximate equation for a cluster and, after rounding off coefficients, the corresponding exact expression. Explicitly, using the approximated orbits, we find:

$$
\begin{aligned}
c_{4,1}(x) & =o_{4,2}(x) \cdot o_{4,3}(x), \\
& \simeq x^{8}-x^{7}-7.000001 x^{6}+6.000004 x^{5}+15 x^{4}-10.00001 x^{3}-10 x^{2}+4 x+1, \\
& =x^{8}-x^{7}-7 x^{6}+6 x^{5}+15 x^{4}-10 x^{3}-10 x^{2}+4 x+1, \quad \Delta=17^{7} .
\end{aligned}
$$

The discriminant $\Delta$ corroborates that Eq. (11) decomposes over $\mathbb{Q}(\sqrt{17})$, as it should. The algebraic character of the coefficients of the three period-four orbits forms two groups, according to the algebraic nature, integer or quadratic, of the roots of

$$
\mathbb{S}_{4}(\sigma)=(\sigma+1)\left(\sigma^{2}-\sigma-4\right),
$$

where $\sigma$ is the sum of the orbital points. When the root $\sigma=-1$ is substituted into the periodfour carrier $\psi_{4}(x)$, defined by Eq. (1) in Ref., 1 namely

$$
\begin{gathered}
\psi_{4}(x)=x^{4}-\sigma x^{3}+\frac{1}{2}\left(\sigma^{2}+\sigma-8\right) x^{2}-\frac{1}{6}\left(\sigma^{3}+3 \sigma^{2}-20 \sigma+2\right) x \\
+\frac{1}{24}(\sigma-3)\left(\sigma^{3}+9 \sigma^{2}-2 \sigma-16\right),
\end{gathered}
$$


one obtains $o_{4,1}(x)$. Substituting $(1-\sqrt{17}) / 2$ and $(1+\sqrt{17}) / 2$ into Eq. (13), roots of the quadratic factor in Eq. (12), we get $o_{4,2}(x)$ and $o_{4,3}(x)$, the pair of orbits conjugated over $\mathbb{Q}(\sqrt{17})$. For details, see Refs $\frac{115}{10}$.

Table 2. The selective factorization of period-four orbits. See text.

\begin{tabular}{|c|c|c|c|c|c|c|c|c|c|c|}
\hline Polynomial & $Q_{15}$ & $Q_{17}$ & $Q_{30}$ & $Q_{34}$ & $Q_{60}$ & $Q_{68}$ & $Q_{120}$ & $Q_{136}$ & $Q_{240}$ & $Q_{272}$ \\
\hline Degree & 8 & 16 & 16 & 32 & 32 & 64 & 64 & 128 & 128 & 256 \\
\hline Orbits & $O_{4,1}$ & $\begin{array}{l}O_{4,2} \\
O_{4,3}\end{array}$ & $o_{4,1}$ & $\begin{array}{l}O_{4,2} \\
O_{4,3}\end{array}$ & $O_{4,1}$ & $\begin{array}{l}O_{4,2} \\
O_{4,3}\end{array}$ & $o_{4,1}$ & $\begin{array}{l}O_{4,2} \\
O_{4,3}\end{array}$ & $o_{4,1}$ & $\begin{array}{l}O_{4,2} \\
O_{4,3}\end{array}$ \\
\hline
\end{tabular}

Table 3. The six period-five orbits in existence for the quadratic map, characterized by one orbital point, and by the sum $\sigma_{5, \ell}$ of its points. The remaining points follow by iterating $x_{t+1}=2-x_{t}^{2}$.

\begin{tabular}{|c||c|c|}
\hline Orbit & $x_{1}$ & $\sigma_{5, \ell}$ \\
\hline$o_{5,1}$ & -1.6825070656623623377 & 1 \\
\hline$o_{5,2}$ & -1.9638573945254134021 & -3.3722813232690143300 \\
$o_{5,3}$ & -1.1601138191423963584 & 2.3722813232690143300 \\
\hline$o_{5,4}$ & -1.9590598825049889879 & -3.0838723594356076658 \\
$o_{5,5}$ & -1.6415268824145526527 & 0.7868018150723329561 \\
$o_{5,6}$ & -1.0579280206539249147 & 3.2970705443632747098 \\
\hline
\end{tabular}

Table 4. The selective factorization of period-five orbits.

\begin{tabular}{|ccccccccccccc|}
\hline$Q_{11}$ & $Q_{22}$ & $Q_{33}$ & $Q_{31}$ & $Q_{44}$ & $Q_{66}$ & $Q_{62}$ & $Q_{88}$ & $Q_{132}$ & $Q_{124}$ & $Q_{176}$ & $Q_{264}$ & $Q_{248}$ \\
\hline 10 & 20 & 20 & 30 & 40 & 40 & 60 & 80 & 80 & 120 & 160 & 160 & 240 \\
\hline$O_{5,1}$ & $O_{5,1}$ & $O_{5,2}$ & $O_{5,4}$ & $O_{5,1}$ & $O_{5,2}$ & $O_{5,4}$ & $O_{5,1}$ & $O_{5,2}$ & $O_{5,4}$ & $O_{5,1}$ & $O_{5,2}$ & $O_{5,4}$ \\
& & $O_{5,3}$ & $O_{5,5}$ & & $O_{5,3}$ & $O_{5,5}$ & & $O_{5,3}$ & $O_{5,5}$ & & $O_{5,3}$ & $O_{5,5}$ \\
& & & $O_{5,6}$ & & & $O_{5,6}$ & & & $O_{5,6}$ & & & $O_{5,6}$ \\
\hline
\end{tabular}

Thus, we see that even modest numerical knowledge of the orbital coefficients can disclose the exact expression of the cluster equation. This procedure is a significant asset when searching for exact expressions for clusters aggregating orbits of high periods: it allows one to profit from approximate numerical information to correctly extract exact equations.

Table 2 shows the $Q_{\ell}(x)$ polynomials that generate period-four orbits for $\ell \leq 400$. The topmost line identifies the polynomials while the second line refers to their degrees. Under them are indicated the orbits $o_{4, j}$ where the zeros of $Q_{\ell}(x)$ land. As illustrated by the highlighting, it is not difficult to recognize that there are two nested sequences of period-four generating $Q_{\ell}(x)$ polynomials, namely

$$
Q_{15 \times 2^{n}}(x) \quad \text { and } \quad Q_{17 \times 2^{n}}(x), \quad n=0,1,2,3, \ldots
$$

Table 2 reveals the following remarkable facts:

i) All three period-four orbits in existence are generated by the zeros of $Q_{\ell}(x)$, in a cyclic manner;

ii) While orbits generated by $Q_{15 \times 2^{n}}(x)$ consistently land on $o_{4,1}(x)$, the orbit with integer coefficients, orbits generated by $Q_{17 \times 2^{n}}(x)$ land either on $o_{4,2}(x)$ or $o_{4,3}(x)$, orbits which have quadratic numbers as coefficients. This means that orbits belonging to the same number field are segregated automatically, filtered, by the zeros of $Q_{\ell}(x)$; 
iii) For a given period $k$, the coefficient of $x^{k-1}$ in the orbital equation is $-\sigma$. This fact provides easy access to the equation defining the sum of the orbital point $\$ 1$ which, in the present example, turns out to given by Eq. (12).

As discussed in the continuation, these three features are found to be generic characteristics of other $Q_{\ell}(x)$ polynomials and periods.

Table 5. Selective factorization of orbits of periods six, seven, and eight.

\begin{tabular}{|c|c|c|c|c|c|c|c|c|c|c|c|c|}
\hline$Q_{13}$ & $Q_{21}$ & $Q_{26}$ & $Q_{42}$ & $Q_{63}$ & $Q_{52}$ & $Q_{65}$ & $Q_{84}$ & $Q_{126}$ & $Q_{104}$ & $Q_{130}$ & $Q_{168}$ & $Q_{252}$ \\
\hline 12 & 12 & 24 & 24 & 36 & 48 & 48 & 48 & 72 & 96 & 96 & 96 & 144 \\
\hline \multirow[t]{4}{*}{$o_{6,1}$} & $o_{6,2}$ & $o_{6,1}$ & $O_{6,2}$ & $o_{6,3}$ & $o_{6,1}$ & $o_{6,6}$ & $o_{6,2}$ & $o_{6,3}$ & $o_{6,1}$ & $o_{6,6}$ & $o_{6,2}$ & $o_{6,3}$ \\
\hline & & & & $O_{6,4}$ & & $o_{6,7}$ & & $o_{6,4}$ & & $o_{6,7}$ & & $o_{6,4}$ \\
\hline & & & & $o_{6,5}$ & & $o_{6,8}$ & & $o_{6,5}$ & & $o_{6,8}$ & & $o_{6,5}$ \\
\hline & & & & & & 06,9 & & & & $O_{6,9}$ & & \\
\hline$Q_{43}$ & $Q_{86}$ & $Q_{129}$ & $Q_{127}$ & $Q_{172}$ & $Q_{258}$ & $Q_{254}$ & $Q_{344}$ & & & & & \\
\hline 42 & 84 & 84 & 126 & 168 & 168 & 252 & 336 & & & & & \\
\hline$o_{7,1}$ & $o_{7,1}$ & $O_{7,4}$ & $o_{7,10}$ & $O_{7,1}$ & $O_{7,4}$ & $o_{7,10}$ & $o_{7,1}$ & & & & & \\
\hline$o_{7,2}$ & $o_{7,2}$ & $o_{7,5}$ & $O_{7,11}$ & $o_{7,2}$ & $o_{7,5}$ & $o_{7,11}$ & $o_{7,2}$ & & & & & \\
\hline \multirow[t]{7}{*}{$o_{7,3}$} & $o_{7,3}$ & $o_{7,6}$ & $o_{7,12}$ & $O_{7,3}$ & $O_{7,6}$ & $o_{7,12}$ & $o_{7,3}$ & & & & & \\
\hline & & $o_{7,7}$ & $o_{7,13}$ & & $o_{7,7}$ & $o_{7,13}$ & & & & & & \\
\hline & & $O_{7,8}$ & $o_{7,14}$ & & 07,8 & 07,14 & & & & & & \\
\hline & & $o_{7,9}$ & $o_{7,15}$ & & $O_{7,9}$ & $o_{7,15}$ & & & & & & \\
\hline & & & $o_{7,16}$ & & & $o_{7,16}$ & & & & & & \\
\hline & & & $o_{7,17}$ & & & $o_{7,17}$ & & & & & & \\
\hline & & & 07,18 & & & $0_{7,18}$ & & & & & & \\
\hline$Q_{51}$ & $Q_{85}$ & $Q_{102}$ & $Q_{170}$ & $Q_{255}$ & $Q_{257}$ & $Q_{340}$ & & & & & & \\
\hline 32 & 64 & 64 & 128 & 128 & 256 & 256 & & & & & & \\
\hline$o_{8,1}$ & $o_{8,3}$ & $o_{8,1}$ & $o_{8,3}$ & $0_{8,7}$ & $o_{8,15}$ & $o_{8,3}$ & & & & & & \\
\hline \multirow[t]{4}{*}{$O_{8,2}$} & 08,4 & $o_{8,2}$ & $0_{8,4}$ & 08,8 & 08,16 & $0_{8,4}$ & & & & & & \\
\hline & $o_{8,5}$ & & $o_{8,5}$ & $O_{8,9}$ & $o_{8,17}$ & $o_{8,5}$ & & & & & & \\
\hline & $o_{8,6}$ & & $o_{8,6}$ & $o_{8,10}$ & $0_{8,18}$ & $o_{8,6}$ & & & & & & \\
\hline & & & & 08,14 & $o_{8,30}$ & & & & & & & \\
\hline
\end{tabular}

\subsection{Preperiodic generation of period five orbits}

For period-five, preperiodic points orderly generate the six orbits recorded in Table 3, where they are characterized by one orbital point as well as by the sum $\sigma_{5, \ell}$ of its five points. The first few $Q_{\ell}(x)$ having roots which land on period-five orbits are recorded in Table 4 . Similarly to Table 2 the topmost line shows the relevant $Q_{\ell}(x)$, with their degrees on the second line. For period-five, the highlighting shows the existence of three distinct nested sequences, namely:

$$
Q_{11 \times 2^{n}}(x), \quad Q_{33 \times 2^{n}}(x), \quad Q_{31 \times 2^{n}}(x), \quad n=0,1,2,3, \ldots
$$

Once again, the $Q_{\ell}(x)$ generate systematically all existing orbits in a cyclic way, and segregate them automatically according to the algebraic nature of the orbital coefficients. As before, from the numerically obtained orbits we get the exact expression defining $\sigma_{5, \ell}$ for the six orbital points, namely

$$
\mathbb{S}_{5}(\sigma)=(\sigma-1)\left(\sigma^{2}+\sigma-8\right)\left(\sigma^{3}-\sigma^{2}-10 \sigma+8\right) .
$$

The three factors composing $\mathbb{S}_{5}(\sigma)$ correspond to the three groups of orbits discriminated in Table 4 
From numerically approximate orbits we obtain exact expressions for the pair of period-five clusters:

$$
\begin{gathered}
c_{5,1}(x)=x^{10}+x^{9}-10 x^{8}-10 x^{7}+34 x^{6}+34 x^{5}-43 x^{4}-43 x^{3}+12 x^{2}+12 x+1, \\
c_{5,2}(x)=x^{15}-x^{14}-14 x^{13}+13 x^{12}+78 x^{11}-66 x^{10}-220 x^{9}+165 x^{8}+330 x^{7} \\
\quad-210 x^{6}-252 x^{5}+126 x^{4}+84 x^{3}-28 x^{2}-8 x+1 .
\end{gathered}
$$

These aggregates factor into quintics over $\mathbb{Q}(\sqrt{33})$ and $\mathbb{Q}(\sqrt[3]{-62+95 \sqrt{-3}})$, respectively, thereby providing exact explicit expressions for the remaining five period-five orbits. Note that a complex number field is needed to extract the three real orbits entangled in $c_{5,2}(x)$.

Once again, apart from $o_{5,1}(x)$, the real projections of the five remaining orbits are necessarily approximated. However, when multiplying together the distinct orbits arising from the roots of a fixed $Q_{\ell}(x)$ polynomial we obtain a cluster whose coefficients turn out to be very close to integers. Rounding them off yields the final exact expression with integer coefficients. Even using modest numerical approximations of the orbital points one can obtain exact cluster equations as may be validated by comparing its roots with numerical values generated by iteration of the equations of motion ${ }^{[1]}$ We have encountered no case where the above procedure failed to produce exact expressions for orbital clusters.

\subsection{Preperiodic generation of periods six, seven, and eight}

Altogether, there are nine orbits of period six, listed in Table 1 of Ref ${ }^{1}$ The orbits form four groups, corresponding to the four factors composing $\mathbb{S}_{6}(\sigma)$, which defines the sum of their orbital points:

$$
\mathbb{S}_{6}(\sigma)=(\sigma+1)(\sigma-1)\left(\sigma^{3}-21 \sigma+28\right)\left(\sigma^{4}+\sigma^{3}-24 \sigma^{2}-4 \sigma+16\right) .
$$

Thus, there are two isolated orbits, denoted by $o_{6,1}(x)$ and $o_{6,2}(x)$, which have integer coefficients, a group of three orbits with cubic coefficients, $o_{6,3}(x), o_{6,4}(x)$, and $o_{6,5}(x)$, and a group of four orbits with quartic coefficients, $o_{6,6}(x), o_{6,7}(x), o_{6,8}(x)$, and $o_{6,9}(x)$.

The $Q_{\ell}(x)$ polynomials generating all period-six orbits are collected in the upper portion of Table 5. The center portion of the table collects the $Q_{\ell}(x)$ polynomials that generate all period-seven orbits. The three factors which compose $\mathbb{S}_{7}(\sigma)$, as well as the procedure to obtain them, valid for any arbitrary period $k$, are given explicitly in Ref ${ }^{1}$ Finally, the lower portion of Table 5 presents $Q_{\ell}(x)$ polynomials for all period-eight orbits. Expressions for $\mathbb{S}_{8}(\sigma), \mathbb{S}_{9}(\sigma)$, and $\mathbb{S}_{10}(\sigma)$ are given in the Appendix. The individual factors composing the several $\mathbb{S}_{k}(\sigma)$ fix the algebraic character of the coefficients for every individual period- $k$ orbit.

\section{Conclusions and outlook}

This paper complements the iterative approach recently discussed in this Journal ${ }^{1]}$ Here, the aim was to obtain an alternative method to extract systematically exact expressions for orbital equations of arbitrary periods of the quadratic map in the partition generating limit. The alternative method consists of using preperiodic points comfortably generated by an infinite family of monogenic ${ }^{9}$ polynomials $Q_{\ell}(x)$ to selectively extract equations of motion, one by one, with no need for iterating polynomials. The procedure is simple to implement and effective.

Both methods, polynomial iteration or preperiodic points, are essentially limited by the capability of the hardware and software used to handle ever growing polynomials with huge numerical coefficients. While this limitation impacts the maximum period accessible to algebraic manipulations, continued advances in computer systems will certainly continue to expand the 
range available to investigate algebraic dynamics $\frac{10}{10}$ exactly, allowing one to advance into new research realms by applying any of the two methodologies now available.

The quadratic map in Eq. (1) offers a number of enticing problems worth pursuing. For $a=0$, the orbits reproduce several of the familiar cyclotomic polynomials. For $a=2$, the infinite set of periodic orbits embedded in the fully developed chaos consists of a cyclotomiclike set of objects that share many properties with the standard cyclotomic polynomials.1 ${ }^{1 / 5}$ The dynamics for other values of $a$, when real and complex orbits coexist, is totally open to investigation. Integer and rational values of $a$ are first good candidates to learn how number towers unfold arithmetically. In particular $a=1$, say, offers the possibility of learning about the interplay of coexisting orbits defined by towers of real and complex algebraic quantities, a new and totally unexplored world.

Table 6. The thirty period-eight orbits, characterized by one orbital point and the sum $\sigma_{8, j}$ of the eight orbital points. Complete orbits may be generated by iterating $x_{t+1}=2-x_{t}^{2}$. The values of $\sigma_{8, j}$ are roots of Eq. A.1.

\begin{tabular}{|c||c|c|}
\hline Orbit & $x_{1}$ & $\sigma_{8, j}$ \\
\hline$o_{8,1}$ & -1.984841019343871516522912 & -2.561552812808830274910705 \\
$o_{8,2}$ & -1.632393824712443381743705 & 1.561552812808830274910705 \\
\hline$o_{8,3}$ & -1.994538346771576098442202 & -4.914223945039180928208247 \\
$o_{8,4}$ & -1.951023935960873278145019 & -2.056133705669804629074231 \\
$o_{8,5}$ & -1.573489876066966494406107 & 2.352671132230350653297542 \\
$o_{8,6}$ & -1.738177892611056635019841 & 3.617686518478634903984936 \\
\hline$o_{8,7}$ & -1.999392903955743234132651 & -9.192789454613742069133553 \\
$o_{8,8}$ & -1.970324466935013007529986 & -3.456817575142370539782937 \\
$o_{8,9}$ & -1.898269888071802444878746 & 0.2899559343985875455768889 \\
$o_{8,10}$ & -1.926986288411966237038570 & 0.5911182259052169094701404 \\
$o_{8,11}$ & -1.687334295667532671943878 & 1.104146442912019886485395 \\
$o_{8,12}$ & -1.784801166495895642936504 & 1.766177771271217083497342 \\
$o_{8,13}$ & -1.224840406098499982124136 & 4.323105719133964018738106 \\
$o_{8,14}$ & -0.9785858338678472056275101 & 5.575102936135107165148616 \\
\hline$o_{8,15}$ & -1.999402315686187320311568 & -9.229152884143427069047449 \\
$o_{8,16}$ & -1.994622984321411050382163 & -4.890484957292577579687509 \\
$o_{8,17}$ & -1.970783422280053272462841 & -3.270791497771093661943518 \\
$o_{8,18}$ & -1.985075746010103467347859 & -2.686687205494066985177061 \\
$o_{8,19}$ & -1.867014790104666658874452 & -2.631302749249868342542760 \\
$o_{8,20}$ & -1.951780177216468660609469 & -2.375150786131144323343685 \\
$o_{8,21}$ & -1.899833775307932554727627 & 0.1093594181979987080920541 \\
$o_{8,22}$ & -1.928111435796830339423029 & 0.7797117117494440466968254 \\
$o_{8,23}$ & -1.637914468949626157934556 & 0.8210383845347267439370462 \\
$o_{8,24}$ & -1.692053315444146323149561 & 1.834669950453959306149210 \\
$o_{8,25}$ & -1.788077312909154517799568 & 1.949372205257687870932170 \\
$o_{8,26}$ & -1.579860384419790809298828 & 2.955978611262725340347639 \\
$o_{8,27}$ & -1.742147511485913313291649 & 3.175217183647612247623081 \\
$o_{8,28}$ & -1.236026775830388280510551 & 3.430438713308632045309287 \\
$o_{8,29}$ & -.9068916550266425682669635 & 4.627714403788193594030641 \\
$o_{8,30}$ & -.9929341328818267363874723 & 6.400069497881198058624029 \\
\hline
\end{tabular}

\section{Acknowledgments}

This work was started during a visit to the Max-Planck Institute for the Physics of Complex Systems, Dresden, gratefully supported by an Advanced Study Group on Forecasting with Lyapunov vectors. The author was partially supported by CNPq, Brazil, grant 304719/2015-3. 


\section{Appendix A. Expressions of $\mathbb{S}_{k}(\sigma)$ for periods $k=8,9$ and 10}

Table 6 defines the thirty period-eight orbits $o_{8, i}(x)$ listed in Table 5 The individual factors which fix the coefficients, and therefore the algebraic nature for all period-eight orbits, are roots $\sigma_{8, j}$ of:

$$
\begin{aligned}
\mathbb{S}_{8}(\sigma)= & \left(\sigma^{2}+\sigma-4\right)\left(\sigma^{4}+\sigma^{3}-23 \sigma^{2}-\sigma+86\right) \times \\
& \left(\sigma^{8}-\sigma^{7}-75 \sigma^{6}+261 \sigma^{5}+474 \sigma^{4}-2764 \sigma^{3}+3560 \sigma^{2}-1696 \sigma+256\right) \times \\
& \left(\sigma^{16}-\sigma^{15}-120 \sigma^{14}+292 \sigma^{13}+4390 \sigma^{12}-13894 \sigma^{11}-66604 \sigma^{10}+257972 \sigma^{9}\right. \\
& \quad+422785 \sigma^{8}-2255633 \sigma^{7}-434628 \sigma^{6}+9169776 \sigma^{5}-6074688 \sigma^{4} \\
& \left.\quad-12553200 \sigma^{3}+18123520 \sigma^{2}-7237376 \sigma+591872\right) .
\end{aligned}
$$

Manifestly, there are four classes of orbital complexity, corresponding to the factors of degrees $2,4,8,16$. See Ref $!^{1}$ for details.

By constructing tables analogous to Table 6 we get expressions for $\mathbb{S}_{k}(\sigma)$ which define the sum of orbital points for periods $k=9$ and 10 , as well as the algebraic nature for all the orbits of these periods. From the $1+1+2+4+6+18+24=56$ period-nine orbits we find:

$$
\begin{aligned}
& \mathbb{S}_{9}(\sigma)=\sigma(\sigma-1)\left(\sigma^{2}+\sigma-14\right)\left(\sigma^{4}-\sigma^{3}-27 \sigma^{2}+41 \sigma+2\right) \times \\
&\left(\sigma^{6}-\right.\left.57 \sigma^{4}+76 \sigma^{3}+684 \sigma^{2}-1824 \sigma+1216\right) \times\left(\sigma^{18}-171 \sigma^{16}+342 \sigma^{15}+9234 \sigma^{14}\right. \\
&-25992 \sigma^{13}-216030 \sigma^{12}+707940 \sigma^{11}+2274813 \sigma^{10}-8209976 \sigma^{9}-9918855 \sigma^{8} \\
&+37877526 \sigma^{7}+24342192 \sigma^{6}-74783088 \sigma^{5}-43931952 \sigma^{4}+53908320 \sigma^{3} \\
&\left.+40777344 \sigma^{2}+2976768 \sigma-622592\right) \times\left(\sigma^{24}+\sigma^{23}-217 \sigma^{22}+175 \sigma^{21}+17702 \sigma^{20}\right. \\
&-34450 \sigma^{19}-713778 \sigma^{18}+1978990 \sigma^{17}+15416541 \sigma^{16}-54321171 \sigma^{15} \\
&-174437381 \sigma^{14}+785332035 \sigma^{13}+859244108 \sigma^{12}-5882602892 \sigma^{11}-234490112 \sigma^{10} \\
&+20915101712 \sigma^{9}-7544198464 \sigma^{8}-35643986496 \sigma^{7}+15193333504 \sigma^{6} \\
&+28736640000 \sigma^{5}-9095376896 \sigma^{4}-11068506112 \sigma^{3}+1392246784 \sigma^{2} \\
&+1681915904 \sigma+134217728) .
\end{aligned}
$$

Apart from a pair of orbits with integer coefficients, there are five classes of orbital complexity, corresponding to the remaining factors in $\mathbb{S}_{9}(\sigma)$, and which define orbital coefficients of algebraic degrees $2,4,6,18$, and 24 .

Analogously, from the $1+2+3+8+15+30+40=99$ period-ten orbits we get the expressions that fix coefficients and the algebraic character for all orbits:

$$
\begin{aligned}
\mathbb{S}_{10}(\sigma)= & \sigma\left(\sigma^{2}-\sigma-10\right)\left(\sigma^{3}+\sigma^{2}-10 \sigma-8\right)\left(\sigma^{8}+\sigma^{7}-79 \sigma^{6}+11 \sigma^{5}+1766 \sigma^{4}-1980 \sigma^{3}\right. \\
& \left.-6120 \sigma^{2}+6400 \sigma+2560\right) \times\left(\sigma^{15}+\sigma^{14}-138 \sigma^{13}+80 \sigma^{12}+6278 \sigma^{11}-13450 \sigma^{10}\right. \\
& -98056 \sigma^{9}+360148 \sigma^{8}+54921 \sigma^{7}-1300271 \sigma^{6}+477210 \sigma^{5}+1783924 \sigma^{4} \\
& \left.-627480 \sigma^{3}-972256 \sigma^{2}+154496 \sigma+141824\right) \times\left(\sigma^{30}-\sigma^{29}-308 \sigma^{28}+988 \sigma^{27}\right. \\
& +35612 \sigma^{26}-165388 \sigma^{25}-2057832 \sigma^{24}+12178568 \sigma^{23}+64943174 \sigma^{22} \\
& -488429574 \sigma^{21}-1123435104 \sigma^{20}+11745093392 \sigma^{19}+9331519964 \sigma^{18} \\
& -180278015100 \sigma^{17}+3569846216 \sigma^{16}+1838668414168 \sigma^{15}-869341730175 \sigma^{14} \\
& -12716885593921 \sigma^{13}+9033287197044 \sigma^{12}+59693364821364 \sigma^{11} \\
& -48003427786304 \sigma^{10}-186012055610544 \sigma^{9}+147967410696768 \sigma^{8} \\
& +364183488569536 \sigma^{7}-259735306624768 \sigma^{6}-399991760098304 \sigma^{5} \\
& +232462373875712 \sigma^{4}+194622659919872 \sigma^{3}-82496776568832 \sigma^{2} \\
& -29669038489600 \sigma+10950019121152) \times\left(\sigma^{40}-410 \sigma^{38}+820 \sigma^{37}+69905 \sigma^{36}\right. \\
& -249444 \sigma^{35}-6473900 \sigma^{34}+32183360 \sigma^{33}+354950530 \sigma^{32}-2315420880 \sigma^{31} \\
& -11590177004 \sigma^{30}+102791354200 \sigma^{29}+201751419530 \sigma^{28}-2950337399160 \sigma^{27} \\
& -577704999440 \sigma^{26}+56031016855856 \sigma^{25}-53560368567875 \sigma^{24}-711891460448400 \sigma^{23} \\
& +1295736491580950 \sigma^{22}+6043257616401300 \sigma^{21}-15792646015357819 \sigma^{20}
\end{aligned}
$$




$$
\begin{aligned}
& -33550951726098500 \sigma^{19}+119952431016405420 \sigma^{18}+113943905945026160 \sigma^{17} \\
& -599301088769483360 \sigma^{16}-185763739671009920 \sigma^{15}+1988903009578851200 \sigma^{14} \\
& -104975203722073600 \sigma^{13}-4318548194918598400 \sigma^{12}+1018330875056000000 \sigma^{11} \\
& +5904641008147348480 \sigma^{10}-1616363148079616000 \sigma^{9}-4743519951800729600 \sigma^{8} \\
& +842331694258585600 \sigma^{7}+1957033680587980800 \sigma^{6}+15775574654976000 \sigma^{5} \\
& -276814761033728000 \sigma^{4}-19653221731532800 \sigma^{3}+11527531161190400 \sigma^{2} \\
& +792421466112000 \sigma-112699941847040) .
\end{aligned}
$$

The above expression shows that there is one period-ten orbit with integer coefficients, and six classes of orbital complexity, each class characterized by coefficients defined by algebraic numbers of degrees $2,3,8,15,30$, and 40 .

Next, there are 186 orbits of period $k=11,335$ of $k=12,630$ of $k=13,1161$ of $k=14,2182$ of $k=15$, etc $\frac{11}{11}$ involving polynomial clusters of degrees 2046,4020,8190,16254, and 32730, respectively. The algebraic properties of the orbits with $k>10$ remain to be investigated. As shown in Refs ${ }^{1 / 5}$, together with $\psi_{k}(x)$, the polynomials $\mathbb{S}_{k}(\sigma)$ form "doublets" defining orbital carriers, which $\sigma$-encode simultaneously all existing period- $k$ orbits and, therefore, contain maximum possible information regarding the complete set of period- $k$ orbits, for any arbitrary period $k$.

\section{References}

1. J.A.C. Gallas, Orbital carriers and inheritance in discrete-time quadratic dynamics, Int. J. Mod. Phys. C 31, 2050100 (2020).

2. J. Argyris, G. Faust, M. Haase, and R. Friedrich, An Exploration of Dynamical Systems and Chaos, Second Edition (Springer, Berlin, 2015).

3. M. Cencini, F. Cecconi, and A. Vulpiani, Chaos - From Simple Models to Complex Systems (World Scientific, Singapore, 2010).

4. M. Ausloos and M. Dirickx (eds.), The Logistic Map: Map and the Route to Chaos: From the Beginning to Modern Applications, Proceedings of the "Verhulst 200 on Chaos", Brussels, Belgium (Springer, Heidelberg, 2005).

5. J.A.C. Gallas, Lasers, stability, and numbers, Physica Scripta 94, 014003 (2019).

6. J.A.C. Gallas, Method for extracting arbitrarily large orbital equations of the Pincherle map, Results in Physics 6, 561-567 (2016).

7. J.A.C. Gallas, Equivalence among orbital equations of polynomial maps, Int. J. Mod. Phys. C 29, 1850082 (2018).

8. S. Pincherle, L'iterazione completa di $x^{2}-2$, Realle Accad. dei Lincei, Rend. della Classe di Scienze Fisiche, Matematiche e Naturali (Roma), Series 5, 29(1), 329-333 (1920).

9. J.A.C. Gallas, Monogenic period equations are cyclotomic polynomials, Int. J. Mod. Phys. C 31, 2050058 (2020).

10. J.H. Silverman, The Arithmetic of Dynamical Systems (Springer, New York, 2007).

11. O.J. Brison and J.A.C. Gallas, What is the effective impact of the explosive orbital growth in discrete-time one-dimensional polynomial dynamical systems? Physica A 410, 313-318 (2014). 